International Mathematical Forum, 1, 2006, no. 21, 1033-1041

\title{
Calculating the eigenstructure of a regular matrix pencil - an approach based on the Weierstrass form
}

\author{
Xian Zhang ${ }^{1,2}$ \\ ${ }^{1}$ Center for Control Theory and Guidance Technology \\ Harbin Institute of Technology, PO Box 416, Harbin, 150001, P. R. China \\ ${ }^{2}$ School of Mathematical Science, Heilongjiang University, Harbin 150080, China \\ E-mail: zhangx663@126.com
}

\begin{abstract}
A new and simple approach for calculating the eigenstructure of a regular matrix pencil is presented. This proposed approach transforms the generalized eigenvalues problem into a usual eigenvalues problem, and it also can be used to calculate the Weierstrass form of a regular matrix pencil.
\end{abstract}

Mathematics Subject Classification: 93B55

Keywords: Eigenstructure; Weierstrass form; Jordan canonical form; Regular matrix pencil.

\section{Introduction}

Many authors discussed the generalized eigenvalues problems in matrix theory, both from the algebraic point of view (see [4] and the references therein) and from the numerical (computational) point of view (see [6] and [8], and the references therein). Sometimes, the generalized eigenvalues problems are also formulated to compute the Kronecker form of a matrix pencil (see [2] and [9], the references therein).

A matrix pencil $s F-G$ is be said to be regular if

$$
\operatorname{det}(s F-G) \neq 0 \text { for some } s \in \mathbf{C} \text {. }
$$

The Kronecker form of a regular matrix pencil is also called the Weierstrass form in some literature (e.g., [7]). Moreover, based on the following two reasons, the generalized eigenvalues problems of regular matrix pencils seem to be more important. 
1. In [4] and [2], it is pointed out that computing the Weierstrass form of a regular pencil is a medium step for computing the Kronecker form of a matrix pencil (possible non-square).

2. The condition (1) (i.e., the regularity of $s F-G$ ) is necessary and sufficient to guarantee the existence and the uniqueness of the solution of the descriptor linear systems in the form

$$
F \dot{x}=G x+B u
$$

where $x$ is the state vector, $u$ is the input vector and $F, G$ and $B$ are known matrices (see [10]). Moreover, the generalized eigenvalues and corresponding eigenvectors of $s F-G$ are important and basic to research (2) (see [7]).

The purpose of the paper is to find a new and simple approach for calculating the generalized eigenvalues and corresponding eigenvectors of a regular matrix pencil. The approach is given by transforming the generalized eigenvalues problem of a regular matrix pencil into a usual eigenvalues problem. Therefore, the paper also shows the relation between the Weierstrass form of a regular matrix pencil and the Jordan canonical form of a constructed matrix, and offers an approach for calculating the Weierstrass form of a regular matrix pencil (for details see Section 4). While the usual eigenvalues problem is well-known and can be solved by different methods. For instance, an elementary transformation method from the algebraic point of view is introduced by Cao and Zhang in [1, Lemma 7.3] and several methods from the numerical point of view are discussed by Golub and Wilkinson in [5]. However, we will prefer to use the elementary transformation method in Section 4 since the whole paper is proceeded according to the algebraic point of view.

The next section formulates the problem to be addressed in the paper. Section 3 solves Problem 1 proposed in Section 2. An illustrative example is worked out in Section 4.

\section{Problem formulation}

The section formulates the problem of calculating the generalized eigenvalues and corresponding eigenvectors of a regular matrix pencil. We first introduce some notations.

For a positive integer $k$, let $\langle k\rangle$ and $\overline{\langle k\rangle}$ be the set $\{1,2, \cdots, k\}$ and $\{1$, $2, \cdots, k, \infty\}$, respectively. For a complex number $s$ and a group of positive integers $a_{1}, \cdots, a_{v}$, we denote by $J\left(s, a_{1}, \cdots, a_{v}\right)$ the matrix

$$
s I+\operatorname{diag}\left(N_{a_{1}}, \cdots, N_{a_{v}}\right)
$$


where the notation $N_{b}$ represents the $b \times b$ Jordan block with zero diagonal elements.

Assume that the eigenvalues of an $n \times n$ regular matrix pencil $s F-G$ are $\lambda_{i}$, $i \in \overline{\langle\mu\rangle}$, where $\lambda_{1}, \cdots, \lambda_{\mu}$ represent the mutually distinct finite eigenvalues and $\lambda_{\infty}$ represents the infinite eigenvalue (Note: $\lambda_{\infty}$ is absent if $F$ is nonsingular). The geometric multiplicity of $\lambda_{i}$ is denoted by $\theta_{i}$, and the lengths of those $\theta_{i}$ chains of generalized eigenvectors with $\lambda_{i}$ are denoted by $\rho_{i j}, j \in\left\langle\theta_{i}\right\rangle$. Then, by [3], there holds $n=\sum_{i \in \overline{\langle\mu\rangle}} \sum_{j=1}^{\theta_{i}} \rho_{i j}$.

For convenience, we give the following definition.

Definition 2.1 Suppose the eigenvalues of the regular matrix pencil sF-G are defined previously. Then the matrices

$$
\operatorname{diag}\left(J\left(\lambda_{1}, \rho_{11}, \cdots, \rho_{1 \theta_{1}}\right), \cdots, J\left(\lambda_{\mu}, \rho_{\mu 1}, \cdots, \rho_{\mu \theta_{\mu}}\right)\right)
$$

and $J\left(0, \rho_{\infty 1}, \cdots, \rho_{\infty \theta_{\infty}}\right)$ are, respectively, called the finite canonical form and the infinite canonical form of $s M-N$.

In regard to the finite canonical form and the infinite canonical form of a regular matrix pencil $s F-G$, the following proposition is well-known.

Proposition 2.2 An $n \times n$ regular matrix pencil sF-G possesses the finite canonical form $J_{f}$ and the infinite canonical form $J_{\infty}$ if and only if there exists a nonsingular matrix $\left[\begin{array}{ll}V_{f} & V_{\infty}\end{array}\right] \in \mathbf{C}^{\mathbf{n} \times \mathbf{n}}$ such that

$$
F V_{f} J_{f}=G V_{f}
$$

and

$$
F V_{\infty}=G V_{\infty} J_{\infty}
$$

In [7], it was also pointed out that, when a nonsingular matrix $\left[\begin{array}{ll}V_{f} & V_{\infty}\end{array}\right] \in$ $\mathbf{C}^{\mathbf{n} \times \mathbf{n}}$ satisfies (3) and (4), columns of the matrices $V_{f}$ and $V_{\infty}$ are, respectively, finite and infinite generalized eigenvectors of the regular matrix pencil $s F-G$. Therefore, we also give the following definition.

Definition 2.3 Suppose an $n \times n$ regular matrix pencil $s F-G$ possesses the finite canonical form $J_{f}$ and the infinite canonical form $J_{\infty}$. Then a matrix $\left[\begin{array}{cc}V_{f} & V_{\infty}\end{array}\right] \in \mathbf{C}^{\mathbf{n} \times \mathbf{n}}$ is called a generalized eigenvector matrix of $s F-G$ relative to $J_{f}$ and $J_{\infty}$ if it is nonsingular and satisfies (3) and (4). In this case, $V_{f}$ is said to be finite and $V_{\infty}$ is said to be infinite. 
Obviously, the finite and infinite canonical forms of a regular matrix pencil are unique in the sense that the order of diagonal blocks is omitted. However, the corresponding generalized eigenvector matrix is not unique. Furthermore, according to the above definitions and proposition, the following two special cases (a) and (b) show that the finite and infinite canonical forms are in touch with the well-known Jordan canonical form, which is the reason for so named finite and infinite canonical forms.

(a) If $F$ is nonsingular, then (4) is absent, which implies that (3) is equivalent to $F^{-1} G=V_{f} J_{f} V_{f}^{-1}$, i.e., $J_{f}$ is the Jordan canonical form of the matrix $F^{-1} G$.

(b) If $G$ is nonsingular and $G^{-1} F$ is nilpotent, then (3) is absent, which implies that (4) is equivalent to $G^{-1} F=V_{\infty} J_{\infty} V_{\infty}^{-1}$, i.e., $J_{\infty}$ is the Jordan canonical form of the matrix $G^{-1} F$.

The finite and infinite canonical forms and a corresponding eigenvector matrix of a regular matrix pencil are usually called its eigenstructure in some literature. The problem of calculating the eigenstructure of a regular matrix pencil can be stated as follows.

Problem 1 (Calculate the eigenstructure of a regular matrix pencil) Given an $n \times n$ regular matrix pencil $s M-N$. Calculate its finite and infinite canonical forms and a corresponding eigenvector matrix.

\section{Main Results}

This section gives a new and simple approach for solving Problem 1 proposed in Section 2. let

In the rest of this paper, denote $\Gamma=\{s \in \mathbf{C} \mid \operatorname{det}(\mathrm{sM}-\mathrm{N})=0\}$ and further

$$
\Omega=\left(s_{0} M-N\right)^{-1} M
$$

where $s_{0} \in \mathbf{C} \backslash \Gamma$ is arbitrary but fixed.

According to Jordan canonical form's theory, it is easy to see that the matrix $\Omega$ possesses the following form.

$$
\Omega=T \operatorname{diag}\left(J, J_{\infty}\right) T^{-1}
$$

with

$$
J=\operatorname{diag}\left(J\left(s_{1}, p_{11}, \cdots, p_{1 q_{1}}\right), \cdots, J\left(s_{l}, p_{l 1}, \cdots, p_{l q_{l}}\right)\right)
$$


and

$$
J_{\infty}=J\left(0, p_{\infty 1}, \cdots, p_{\infty q_{\infty}}\right)
$$

where $T \in \mathbf{C}^{\mathbf{n} \times \mathbf{n}}$ is nonsingular, $s_{i} \in \mathbf{C} \backslash\{\mathbf{0}\}$ for any $i \in\langle l\rangle$, and $s_{1}, \cdots, s_{l}$ are mutually distinct.

In order to solve Problem 1, we first prove the following lemma.

Lemma 3.1 Given the matrix $J$ in the form of (7). Further, let

$$
J_{f}=\operatorname{diag}\left(J\left(s_{0}-\frac{1}{s_{1}}, p_{11}, \cdots, p_{1 q_{1}}\right), \cdots, J\left(s_{0}-\frac{1}{s_{l}}, p_{l 1}, \cdots, p_{l q_{l}}\right)\right) .
$$

Then $J$ is the Jordan canonical form of the matrix $\left(s_{0} I-J_{f}\right)^{-1}$, i.e., there exists a nonsingular matrix $Q_{f}$ such that

$$
\left(s_{0} I-J_{f}\right)^{-1}=Q_{f} J Q_{f}^{-1} .
$$

Proof It follows from (9) that

$$
\begin{gathered}
\left(s_{0} I-J_{f}\right)^{-1} \\
=\operatorname{diag}\left(\left[-J\left(-\frac{1}{s_{1}}, p_{11}, \cdots, p_{1 q_{1}}\right)\right]^{-1}, \cdots,\left[-J\left(-\frac{1}{s_{l}}, p_{l 1}, \cdots, p_{l q_{l}}\right)^{-1}\right]\right) .
\end{gathered}
$$

Note that, for any $j \in\left\langle q_{i}\right\rangle$ and $i \in\langle l\rangle$, there holds

$$
\left[-\left(-\frac{1}{s_{i}}+N_{p_{i j}}\right)\right]^{-1}=\left(\frac{1}{s_{i}}-N_{p_{i j}}\right)^{-1}=s_{i} I+\sum_{k=1}^{p_{i j}-1} s_{i}^{k+1} N_{p_{i j}}^{k} .
$$

From which, the matrix $\left[-\left(-\frac{1}{s_{i}}+N_{p_{i j}}\right)\right]^{-1}$ has the unique eigenvalue $s_{i}$ of the algebraic multiplicity $p_{i j}$ and the geometric multiplicity 1 . Therefore, there exists a nonsingular matrix $Q_{i j}$ such that

$$
Q_{i j}^{-1}\left[-\left(-\frac{1}{s_{i}}+N_{p_{i j}}\right)\right]^{-1} Q_{i j}=s_{i}+N_{p_{i j}} .
$$

Let

$$
\left\{\begin{array}{l}
Q_{f}=\operatorname{diag}\left(Q_{1}, \cdots, Q_{l}\right) \\
Q_{i}=\operatorname{diag}\left(Q_{i 1}, \cdots, Q_{i q_{i}}\right), \forall i \in\langle l\rangle
\end{array} .\right.
$$

Then, by (12) and (11), it is easily verified that (10) holds.

By a similar argument to Lemma 3.1, we have 
Lemma 3.2 Given the matrix $J_{\infty}$ in the form of (8). Then $J_{\infty}$ is the Jordan canonical form of the matrix $J_{\infty}\left(s_{0} J_{\infty}-I\right)^{-1}$, i.e., there exists a nonsingular matrix $Q_{\infty}$ such that

$$
J_{\infty}\left(s_{0} J_{\infty}-I\right)^{-1}=Q_{\infty} J_{\infty} Q_{\infty}^{-1}
$$

Based on the above statement, the main result of this paper can be obtained as follows.

theorem 3.3 Given an $n \times n$ regular matrix pencil $s M-N$. Further, assume that $T, Q_{f}, Q_{\infty}, J_{f}$ and $J_{\infty}$ are defined previously, and let

$$
V=T \operatorname{diag}\left(Q_{f}^{-1}, Q_{\infty}^{-1}\right) .
$$

Then the regular matrix pencil $s M-N$ possesses the finite canonical form $J_{f}$, the infinite canonical form $J_{\infty}$ and a corresponding generalized eigenvector matrix $V$.

\section{Proof Let}

$$
T=\left[\begin{array}{ll}
T_{f} & T_{\infty}
\end{array}\right]
$$

where $T_{f}$ and $T_{\infty}$ have the appropriate sizes. Then, by (6), there hold $\Omega T_{f}=$ $T_{f} J$ and $\Omega T_{\infty}=T_{\infty} J_{\infty}$. This, together with Lemmas 3.1 and 3.2, gives

$$
\Omega T_{f} Q_{f}^{-1}=T_{f} Q_{f}^{-1}\left(s_{0} I-J_{f}\right)^{-1}, \Omega T_{\infty} Q_{\infty}^{-1}=T_{\infty} Q_{\infty}^{-1} J_{\infty}\left(s_{0} J_{\infty}-I\right)^{-1},
$$

and hence

$$
M T_{f} Q_{f}^{-1} J_{f}=N T_{f} Q_{f}^{-1}, M T_{\infty} Q_{\infty}^{-1}=N T_{\infty} Q_{\infty}^{-1} J_{\infty}
$$

The nonsingularity of $V$ follows from (14) since $T, Q_{f}^{-1}$ and $Q_{\infty}^{-1}$ are nonsingular. Furthermore, it follows from (15) and (14) that $V=\left[\begin{array}{cc}T_{f} Q_{f}^{-1} & T_{\infty} Q_{\infty}^{-1}\end{array}\right]$. This, together with (16) and Proposition 2.2, completes the proof.

Obviously, Theorem 3.3 gives an approach to calculating the eigenstructure of a regular matrix pencil. In order to use conveniently the approach, we also write it in the form of the following algorithm.

\section{Algorithm [Calculate the eigenstructure of a regular matrix pencil]}

Input An $n \times n$ regular matrix pencil $s M-N$.

Output The finite canonical form $J_{f}$, the infinite canonical form $J_{\infty}$ and a corresponding eigenvector matrix $V$ of $s M-N$.

Step 1 Find a complex number $s_{0}$ satisfying $s_{0} \in \mathbf{C} \backslash \Gamma$. 
Step 2 Calculate the matrix $\Omega$ according to (5).

Step 3 Find a pair of matrices $J$ and $J_{\infty}$ in the form of (7) and (8), respectively, and a nonsingular matrix $T$ satisfying (6).

Step 4 Construct the matrix $J_{f}$ according to (9).

Step 5 Find a pair of nonsingular matrices $Q_{f}$ and $Q_{\infty}$ satisfying (10) and (13), respectively.

Step 6 Calculate the matrix $V$ according to (14).

Finally, we remark the proposed approach as follows.

Firstly, noting that the set $\Gamma$ is finite, we can obtain $s_{0}$ satisfying $s_{0} \in$ $C \backslash \Gamma$ by a test method, and hence the matrix $\Omega$ can be easily obtained. This, together with the above algorithm, implies that, to solve Problem 1, it suffices to compute the matrices $T, J, J_{\infty}, Q_{f}$ and $Q_{\infty}$. According to their definitions, this is transformed to calculate the Jordan canonical forms of three matrices, which can be completed by using the elementary transformation method introduced in [1, Lemma 7.3]. In particular, the matrices $Q_{f}$ and $Q_{\infty}$ may also be directly observed when the diagonal blocks of $J_{f}$ and $J_{\infty}$ have low dimensions (see Section 4).

Secondly, let the matrices $J_{f}, J_{\infty}$ and $V$ be obtained by the above algorithm and denote

$$
W=\left[\begin{array}{ll}
M V_{f} & N V_{\infty}
\end{array}\right]
$$

where $V_{f}$ and $V_{\infty}$ are defined by $\left[\begin{array}{ll}V_{f} & V_{\infty}\end{array}\right]=V$. Then, according to an argument presented in [7, p.10], there holds

$$
W^{-1}(s M-N) V=\operatorname{diag}\left(s I-J_{f}, s J_{\infty}-I\right),
$$

which is the well-known Weierstrass form of the matrix pencil $s M-N$. Therefore, the proposed approach can also used to compute the Weierstrass form of a regular matrix pencil.

Thirdly, it is clear that the proposed approach can calculate the finite and infinite canonical forms and only a corresponding eigenvector matrix. When all (several) corresponding eigenvector matrices are required, one may first calculate the finite and infinite canonical forms using the method given in the paper, and then calculate all the corresponding eigenvector matrices using an approach proposed by the author and its co-authors in [11]. 


\section{Example}

Consider the regular matrix pencil $s M-N$ with coefficient matrices

$$
M=\left[\begin{array}{rrrrrrr}
1 & 0 & 0 & 0 & 0 & 0 & 0 \\
0 & -1 & 0 & -1 & 2 & 0 & 0 \\
0 & 0 & 1 & 2 & 0 & 0 & 1 \\
1 & 0 & 0 & 0 & 0 & 0 & 0 \\
0 & 0 & 0 & 0 & 0 & 0 & 0 \\
0 & 0 & 3 & 0 & 0 & 0 & 0 \\
0 & 0 & -1 & 0 & 0 & 0 & 0
\end{array}\right], N=\left[\begin{array}{rrrrrrr}
0 & 0 & -2 & 0 & 3 & 0 & 0 \\
0 & 3 & 0 & 0 & -2 & 0 & 0 \\
0 & 0 & 1 & 2 & 0 & 0 & 1 \\
1 & 0 & 0 & 0 & 0 & -1 & 0 \\
0 & -1 & 0 & 0 & 1 & 0 & 0 \\
0 & 0 & 3 & 0 & 0 & 0 & -3 \\
0 & 0 & 0 & 1 & 0 & 0 & 0
\end{array}\right] .
$$

Following the steps in Algorithm, we have

Step 1: $s_{0}=2$.

Step 2:

$$
\Omega=\left[\begin{array}{rrrrrrr}
\frac{1}{2} & -\frac{3}{2} & -\frac{1}{2} & 2 & 3 & 0 & \frac{7}{4} \\
0 & -1 & 0 & 1 & 2 & 0 & 1 \\
0 & 0 & \frac{1}{2} & -\frac{1}{2} & 0 & 0 & -\frac{1}{4} \\
0 & 0 & 0 & 1 & 0 & 0 & \frac{1}{2} \\
0 & -1 & 0 & 1 & 2 & 0 & 1 \\
\frac{1}{2} & \frac{3}{2} & \frac{1}{2} & -2 & -3 & 0 & -\frac{7}{4} \\
0 & 0 & \frac{1}{2} & \frac{1}{2} & 0 & 0 & \frac{1}{4}
\end{array}\right]
$$

Step 3: $J=\operatorname{diag}\left(J\left(\frac{3}{4}, 1\right), J(1,2), J\left(\frac{1}{2}, 1\right)\right), J_{\infty}=J(0,1,1,1)$ and

$$
T=\left[\begin{array}{ccccccc}
-\frac{3}{4} & -4 & -\frac{44}{3} & 1 & 0 & 0 & 0 \\
-\frac{1}{3} & -\frac{4}{3} & -\frac{16}{3} & 0 & -1 & -\frac{4}{3} & 3 \\
-\frac{1}{4} & 0 & \frac{4}{3} & 0 & 0 & 0 & 0 \\
\frac{1}{6} & 0 & -\frac{4}{3} & 0 & -1 & -\frac{4}{3} & 1 \\
-\frac{1}{3} & -\frac{4}{3} & -\frac{16}{3} & 0 & -1 & -\frac{4}{3} & 2 \\
-\frac{1}{4} & 0 & 4 & 1 & \frac{21}{4} & 8 & 0 \\
-\frac{1}{12} & 0 & 0 & 0 & 2 & \frac{8}{3} & -2
\end{array}\right]
$$

Step 4: $J_{f}=\operatorname{diag}\left(J\left(\frac{2}{3}, 1\right), J(1,2), J(0,1)\right)$.

Step 5: $Q_{f}=I, Q_{\infty}=I$.

Step 6: $V=T$.

\section{Conclusions}

We present a new and simple method for calculating the generalized eigenstructure of a regular matrix pencil. The proposed approach also shows the 
relation between the generalized eigenvalues problem of a regular matrix pencil and the usual eigenvalues problem of a constructed matrix, and can be used to compute the Weierstrass form of a regular matrix pencil.

ACKNOWLEDGEMENTS. This work was supported in part by the Chinese Natural Science Foundation under Grant No. 10271021 and the Fund of Heilongjiang Education Committee for Overseas Scholars under Grant No. $1054 \mathrm{HQ} 004$.

\section{References}

[1] C. G. Cao and X. Zhang, A series of lecture on Linear Algebra's Methods (in Chinese), Harbin Press, China, 2001.

[2] P. V. Dooren, The computation of Kronecker's canonical form of a singular pencil, Linear Algebra Appl. 27 (1979) 103-140.

[3] M. M. Fahmy and J. O'Reilly, Parametric eigenstructure assignment for continuous-time descriptor systems, Int. J. Control 49 (1989) 129-143.

[4] F. R. Gantmacher, The Theory of Matrices, Chelsea Publishing Company, New York, 1959.

[5] G. H. Golub and J. H. Wilkinson, Ill-conditioned eigensystems and the computation of the Jordan canonical form, SIAM Review 18(4) (1976) 578-619.

[6] G. H. Golub and Q. Ye, An inverse free preconditioned Krylov subspace method for symmetric generalized eigenvalue problems, SIAM J. Sci. Comput. 24 (1) (2002) 312-334.

[7] F. L. Lewis, A survey of linear singular systems, Circuits Systems Signal Process 5 (1986) 3-36.

[8] J. H. Wilkinson, The Algebra Eigenvalue problems, Clarendon, Oxford, 1965.

[9] J. H. Wilkinson, Kronecker's canonical form and the QZ algorithm, Linear Algebra Appl. 28 (1979) 285-303.

[10] E. L. Yip and R. F. Sincovec, Solvability, controllability, and observability of continous descriptor systems, IEEE Tran. Automat. Contr. 26 (1981) 702-707.

[11] X. Zhang, S. Thompson and G.-R. Duan, Full-column Rank Solutions of the Matrix Equation $A V=E V J$, Appl. Math. Comput. 151 (2004), 815-826.

Received: October 9, 2005 